\title{
Learn Street Skateboarding through 3D Simulations of Angle Rotations
}

\author{
Erwin Adi, I Gde Made Krisna Aditya, and Meriyana Citrawati \\ BINUS International - BINUS University, Jakarta, Indonesia
}

\section{eadi@binus.edu; ditz.hj7@gmail.com; m citrawati@yahoo.com \\ Executive Summary}

Learning physical activities such as sports and games is expensive and time-consuming. A common advice is "repetition makes perfection," which implies that wrong actions must soon be noticed and avoided. A knowledgeable tutor is often required to provide good feedback for that purpose. However, this facility is available only for those who can afford tutorial fees and time arrangements must be made well in advance.

Books and videos are often treated as a more affordable media for learning. However, books and videos show a two dimension picture, while the audience might like to see how a particular move is performed from another angle. Quite often, books and videos do not show the picture that its audience would like to visualize. For example, one skateboarding trick is actually best viewed from the back in order to clearly show how it is done; however, most two dimension pictures show the trick from the front where the result of the trick is nicely shown.

A computer software application allows a user to see an object through a three dimensional experience by rotating the object. We adopt this technique to introduce a 3D medium that allows its user to view how skateboarding tricks are done. Specifically, we implement a 3D simulation software application that teaches the fundamental skills involved in street skateboarding. The software provides a high degree of interactivity by allowing users to view a single animated object from different camera angles. A user can interact with the software through a few easy-tounderstand buttons in order to view the picture from other angles. User test results show that using the software is a more effective means to learn skateboarding compared to watching a video demonstration.

A further benefit of this 3D simulation approach is that it facilitates faster video development time by allowing the use of reusable modules for similar movement patterns. Hence the 3D simulation application could be used to learn other sports and games, since most of them show a repeated hand-stroke, leg movement, or jumping posture. The approach discussed in this paper opens a venue to learn sports and games in a more interactive, efficient, and cost-effective way.

Material published as part of this publication, either on-line or in print, is copyrighted by the Informing Science Institute. Permission to make digital or paper copy of part or all of these works for personal or classroom use is granted without fee provided that the copies are not made or distributed for profit or commercial advantage AND that copies 1) bear this notice in full and 2) give the full citation on the first page. It is permissible to abstract these works so long as credit is given. To copy in all other cases or to republish or to post on a server or to redistribute to lists requires specific permission and payment of a fee. Contact 0HPublisher@InformingScience.org to request redistribution permission.
Keywords: Interactivity, 3D simulation, learning, reusable pattern. 


\section{Introduction}

In a recent survey in the United States, skateboarding was found to be the third most popular sport among teenagers, after football and basketball (Wysaski, n.d.). Skateboarding has grown in popularity over the last few years but there remain a number of people who do not know how to skateboard. Moreover, for the more advanced skateboarder, learning tricks is facilitated by watching other people skateboarding, i.e., either in real time or from recorded videos.

All skateboarding tricks were invented by enthusiasts who desired to build a repertoire of tricks and develop a particular skating style of their own. There are countless tricks in skateboarding; an example of a well-known one is the 'Ollie'. The Ollie was invented by Alan Gelfand. He would slam his back foot down on the tail of his board and jump, launching himself and the board into the air and land smoothly on his board again (Figure 1).

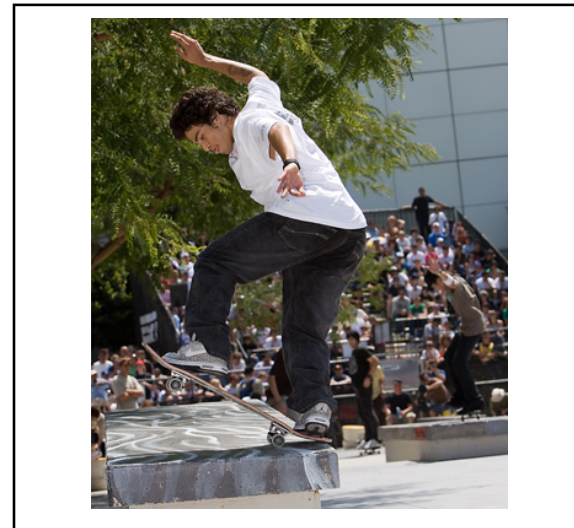

Figure 1: A skateboarding trick

For spectators and beginners, the Ollie can be difficult to imitate. A question which may come to their mind is "How on earth do they do that?" Modern skateboarders seem to defy the laws of physics - but the truth is they use the very same laws to their advantage. Moreover, physical laws such as centripetal force, rotational inertia, or work are far from their minds when they design and execute these 'tricks'. Skateboarders use their inherent talents, most of which stem from long hours of practice. Perfect execution entails even more hours of practice. Therefore, it can be said that the better the tricks are performed and executed, the longer the hours that are poured into mastering the moves.

It came as no surprise to the authors to know that filming a skateboarding video could take almost a full day. A perfect execution is comprised of a combination of extreme tricks and, more often than not, requires at least 10 trials. In brief, learning skateboarding is about practicing the tricks repeatedly.

Furthermore, we observed that skateboarders learn their tricks by looking at how other skateboarders do their moves. They learn from fellow skateboarders who are usually friends because it is the most feasible way to do so. In the absence of a superior to learn from, beginners improve and invent new tricks by watching videos and/or searching the Internet for tips and tricks and attempting to apply them thereafter. If mastering the trick remains a challenge, the only way to learn is by looking at it or watching another skateboarder.

This study seeks to provide an inventive and effective way to learn skateboarding through an interactive 3D simulation.

The remainder of this paper is outlined as follows: Firstly, the current problem encountered by learning skateboarding through available means is surveyed and a proposed solution is presented. Secondly, an existing medium for the proposed solution is examined. Finally, the paper discusses how to design, implement, and test the proposed solution.

\section{Problem Description}

The traditional way of learning skateboarding entails the giving of basic trick instructions by using 2D drawings. Each step of a trick shows foot movement while the skateboarder is in the air. This method could be useful in learning the trick but lacks important details such as the stance and the foot position on the board. Figure 2 is an illustration of the method. 


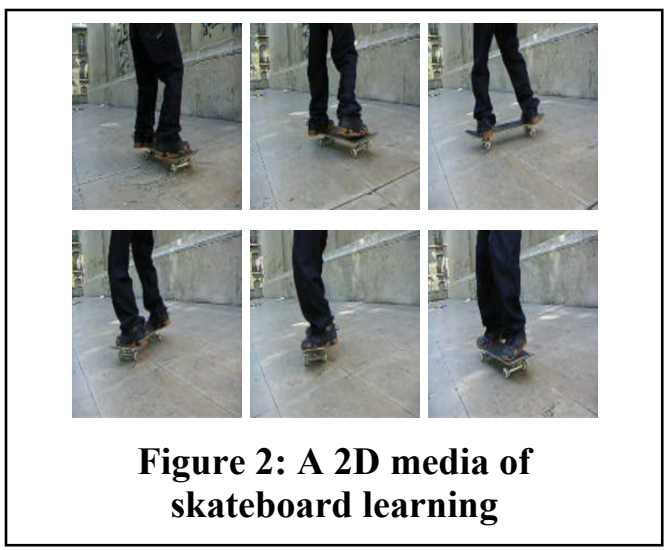

Unfortunately, learning from available means like skateboarder videos or short films broadcast over the Internet, e.g., on YouTube, can sometimes prove to be insufficient as skateboarders will naturally have difficulties, which may be different from each other. Some inexperienced skateboarders may even find themselves unable to appreciate the technique presented in video format including its execution.

We have observed that some people assume that they can learn different tricks by playing skateboard video games on consoles. This observation is particularly true in terms of timing, i.e., deciding exactly when to kick and jump. Learning timing from video games may be ineffective as a way to master the real activity because games are executed using the joystick where the tricks are accomplished by pushing buttons.

\section{Proposed Solutions}

\section{A Survey to Find an Alternative Solution}

To gain insight on how skateboarders learn and to find out about the effectiveness of learning methods with respect to skateboarding, two of the authors went to the Base Skate Park in Bali to conduct a simple survey. Questions in the survey were meant to collect information on who the target audience was, such as age and gender, and what alternative methodology would give a favorable learning experience. The survey was distributed randomly to the target population, and those that returned the survey were interviewed individually.

There were 30 respondents that returned the survey, of which $90 \%$ were male and $10 \%$ were female. $83 \%$ of the respondents were between 15 to 25 years of age. This age range can be categorized as Generation Y which has a strong spending power (Solomon, 2004); however, it is our intention to provide a solution that can be obtained for free.

After further interviewing the respondents individually, it appeared that some answers from the survey sheet should have been filtered out. For example, someone stated that he learned from magazines. On the other hand, his explanation about his learning experience was inconsistent when telling whether the image was a moving or a still object. This made the researchers suspicious about the respondents' ability to understand the differences between the available means of learning skateboarding. Other hurdles included our inability to tell what means was preferred by a respondent when he or she reported to having learned from all options. These difficulties arose mainly because of gaps in levels of education, language, and culture between the researchers and the respondents.

As many as 15 answers covered how the respondents learned skateboarding. $80 \%$ of the respondents learned the sport from friends, while 13\% learned from videos, and $7 \%$ from magazines. This may indicate that skateboarding is a social activity, and may imply that it is more fun to learn directly from peers. On the other hand, it could indicate that the less interactive the medium of learning skateboarding is, the less attractive it becomes as a learning tool. An encouraging 93\% of the respondents said that it would be useful if there existed an interactive 3D simulation software application to learn street skateboarding.

$67 \%$ out of 15 people considered themselves beginners, while $20 \%$ said they were amateurs, and $14 \%$ reported they were professionals. The rest of the 15 skateboarders out of the 30 that returned 
the questionnaire did not fill in this part of the survey. It could be assumed that these respondents were either beginners or amateurs and did not know the difference between the criteria. Whether we would consider this assumption or not, there is sufficient confidence that a product to teach beginners and amateurs instead of professionals would cover most of the population of skateboarders.

In addition to the above observation, the authors interviewed a professional skateboarder, Firman Boesly from Indonesia. One controversial, or perhaps, important revelation during that interview was that some tricks are actually best viewed from the back. He explained that most skateboarding videos capture tricks from wrong angles.

\section{An Interactive 3D Demonstrator}

At first, Boesly referred to a Playstation 3 video game called "Skate" (http://skate.ea.com). The game is the best skateboarding game available on the market according to reviewers (Cave, n.d.). The player controls the character by maneuvering the two game-controller sticks - one stick controls the body, while the other controls the legs. Critics said that the game was hard to master, but proponents argued that the hard combination of the sticks was what made the play fun and rewarding.

For the skateboarders, the game offered a new medium which they could learn from. For per-

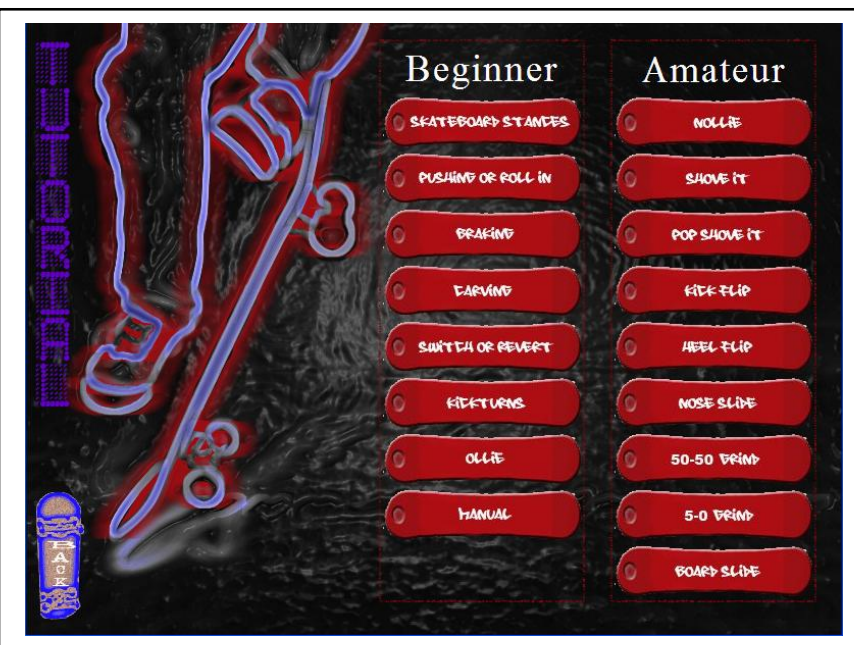

Figure 3: Simple interface to show a chosen trick forming the Ollie trick, a player could move the controller stick back and forth. The game was able to show through a moving $3 \mathrm{D}$ image how the trick was performed. However, the player must master a range of complicated combinations of flicking the controls to view some further tricks.

Boesly was excited to hear our proposal about having a similar 3D animation that could show each of the popular tricks through clicking a single button. A menu was easily constructed through an interface displayed in Figure 3. A 3D simulation software application was developed to show the tricks from different angles, in re-

sponse to Boesly's opinion that some tricks were better viewed from other uncommon shots such as from the back.

We proposed to build the 3D simulation software as a proof of the above concept.

\section{Existing Simulation Software and Their Effectiveness on Learning}

We searched for any existing solution and investigated the effectiveness of 3D simulation software for learning purposes. Simulation could be attributed to a range of meanings from a physical prototype to a video game (Greasley, 2008, p. 1). Based on relevance, simulation software may be categorized into:

- Science and engineering software. The software does not always show animation, but rather yields numbers as the product. For example, cardiovascular assist devices are ma- 
thematically tested through a simulation software application to measure the pressure, flow, pipe dimensions, etc. prior to animal and clinical testing (Gregory, Greatrex, Timms, Gaddum, Pearcy, \& Fraser, 2010).

- Business and manufacturing software, as assessed by Klassen and Willoughby (2003). Examples are listed in Reed Business Information (2009).

- Simulation game "in which participants are provided with a simulated environment in which to play" (Cruickshank, 1980 in Connolly \& Stansfield, 2006).

There is no clear wall that separates the above categories, especially when one examines its effectiveness on enhancing learning. Science and engineering simulation software plays an important part in many businesses; likewise, game-based models are also used to improve the effectiveness of people in the industries (Aldrich, 2005).

Industry players have little doubt on the effectiveness of simulation software. In his book, Greasley (2008) revealed that $41.67 \%$ of surveyed respondents in one study believed that the results of a simulation study would actually lead to change, while $25 \%$ of them stated otherwise. The latter might have not known of the benefits, including how to apply the techniques.

Furthermore, an experiment showed that $55 \%$ of engineer respondents had a more complex design plan after playing a related design-engineering game (Shaffer, 2006). The engineers considered $47 \%$ more features while making a decision.

How video games have positive use to promote knowledge in sport players has been studied by Goodman, Bradley, Paras, Williamson, and Bizzochi (2005). They revealed that youth hockey players who played "the experimental version of the video game scored significantly higher on a concussion symptoms questionnaire, in a significantly faster time, than participants playing the control version of the game." Their study made use of animated icons for the players to stack and match the correct concussion symptoms (e.g., seeing stars).

\section{Game-based Simulation Software for Sport}

Out of the abundant game genres available in the market, this paper studied those that are sportsrelated for the purpose of understanding where the challenges of skateboarding simulations are. At the time of writing, a naïve Google search on a keyword "simulation game" returns a bit more than 20 million results, whereas a search on a keyword "simulation game skateboarding" returns about $1 \%$ of it. It was not a surprise that most sports-related games are related to the many people that play them in real life, such as those listed in HighwayHome (n.d.). Some highly popular sports such as golf, hockey, basketball, etc. enjoy a sophisticated indoor video simulator as can be seen in Figure 4 (http://www.athletiquest.com/indoor-simulators.php), while there are other more affordable simulators for other sports games such as those provided by Nintendo Wii (http://www.nintendo.com/wii). 


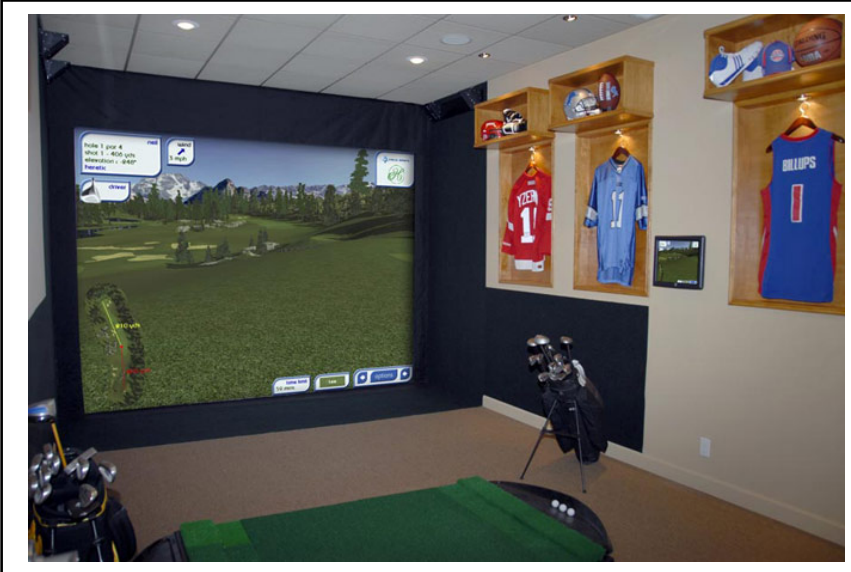

Figure 4: Indoor golf simulator

At the time of the writing, we found only two products that are related to a skateboarding simulator. One is depicted in Figure 5 (PrimeTime Amusements, n.d.). However it can be seen that the hardware is suitable for arcade businesses and not for home use.

Most of the solutions mentioned are not free since they require hardware or at least sensors to catch and learn the player's motion. There exists a free solution in a form of simulation software application provided by Paperskate and is accessible through a web browser (http://www.paperskate3d.com). One could learn about how a skateboard trick begins and ends from any point of view or angle by interacting through mouse and keyboard. The website receives many praises from reviewers. However, the solution only shows how a skateboard is positioned for doing the tricks; it does not show how a skilled instructor initiates the trick such as slamming his back foot.

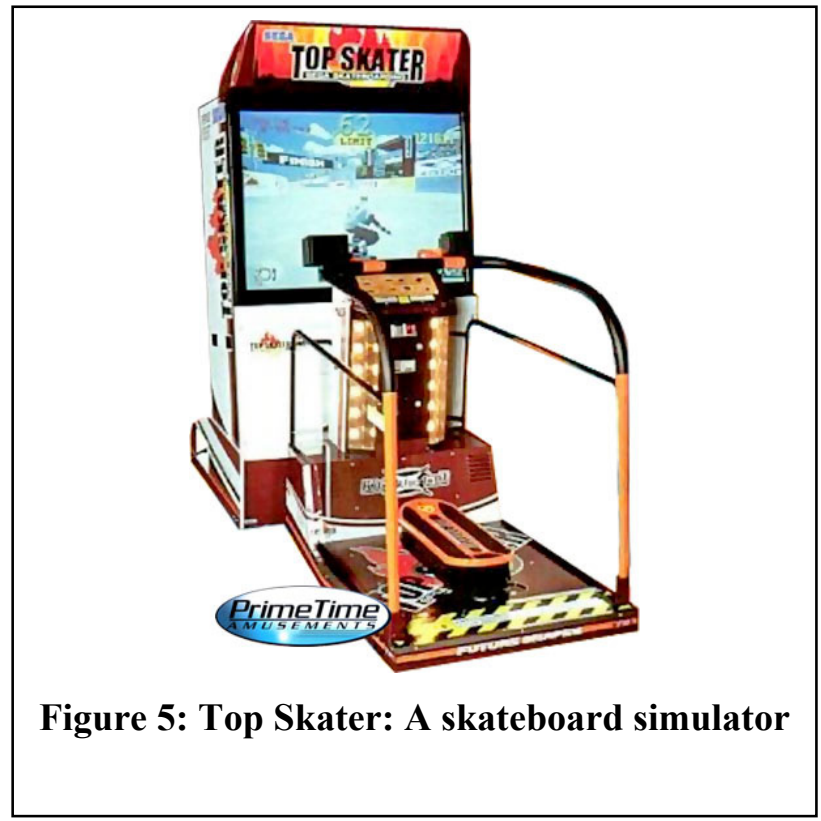




\section{Design Considerations}

In light of providing a medium for learning purposes, we present a proof-of-concept software simulator that demonstrates a skilled instructor. It is more feasible to distribute software with a very low or no cost at all, than to distribute the hardware described in the previous section. Software is so portable that it could be transported through the Internet. Hence, we propose to design and use a software application that is interactive and opens up a new experience for learning, taking inspiration from pre-existing skateboarding games.

The software serves as an integrated learning source that could make onlookers and beginners understand skateboarding terms and skateboarding tricks and teach them how to apply the tricks depending on their learning curves. In brief, the aim of the software implementation is to bundle the knowledge that beginners and amateurs need.

Therefore this study proposes the implementation of a Street Skateboarding Simulation: An animated 3D model which creates a skateboarder model that executes skateboarding tricks and provides assistance to beginners. One notable advantage of 3D models over conventional media is their ability to view the same event from a variety of angles.

\section{Skateboarding Styles}

Although there are many skateboarding styles, basically there are two types of styles and stands that are open to difficult tricks. Vert skateboarding is where people skateboard on a curved halfpipe sized ramp, and Street skateboarding is where they skateboard using obstacles available on streets. Because of their potential harm to passersby, street skateboarding activities have been assigned exclusive areas in some locales. Sponsors that host skateboarding competitions mostly exhibit these two styles. Other types of styles may include Freestyle, Transition, Park, Cruising, Technical Flatground, Downhill, and Big Air.

The first two skateboarding styles require willingness and practice for anybody to master. Mostly the learners need a tutor. Hence it was part of our design considerations to select one of these styles to model the tricks. As one of the authors is more knowledgeable in street skateboarding and has a network of street skateboarders, this study designed the 3D animation based on street skateboarding tricks.

\section{Modeling Tools}

There exists a range of 3D Computer Graphics Programs which can be used for modeling, rigging, and animating tasks. In particular, three software packages are widely used. The succeeding paragraphs feature these three programs and briefly discuss what they offer in relation to the building of character movement and the environment for this project.

\section{3ds Max}

$3 \mathrm{ds}$ Max is the most widely used 3D computer graphic and animation program according to the Roncarelli report (Autodesk 3ds Max, n.d.). It has strong modeling capabilities, a flexible plug-in architecture, and a long heritage on the Microsoft Windows platform. Discreet's 3ds Max presents all of its modeling and layout functions in one user interface. $3 \mathrm{ds}$ Max controls are iconic, i.e., the buttons appear as icons which represent their functions. The program can create a movie file format or an animation sequence of still frames. Rendering output can also be directed to compressed formats such as AVI and Quicktime MOV using any one of several codecs bundled in the software. 
The workflow of this project in 3ds Max would involve creating objects or imported geometry, adding lights and cameras, enhancing objects with materials and effects, building animation with key frames, animating cameras, and rendering and saving image sequences to other programs.

\section{Maya}

Maya is a high-end 3D computer graphics and 3D modeling software tool mostly used for computer and video games, and video effects in Hollywood (Autodesk Maya, n.d.). It is considered by many professional animators to be the best animation program for choreographing characters. It is a sophisticated piece of software to use since its openness to third party software is capable of changing the standard appearance into a highly customized version of the software.

Maya Embedded Language (MEL) is a scripting language in Maya which serves not only as a scripting language but also as a means to customize Maya's core functionality. Moreover, in Maya, user communication is implemented and recorded as a MEL scripting code which can be stored onto a toolbar. For animators who have no experience in $\mathrm{C}$ or $\mathrm{C}++$ programming and compilers, a software development kit is provided.

The workflow for this project in Maya would involve creating scene objects or importing geometry, adding light and cameras, enhancing objects with Hypershade, building animation with key frames, enhancing animation with particles and soft-body dynamics, adding motion controllers and constraints, enhancing animation with fluid effects, animating cameras, and rendering and saving image sequences.

\section{LightWave 3D}

LightWave is a computer graphics program for 3D modeling, animating, and rendering which has a unique user interface (Lightwave 3D, n.d.). Instead of icons, all functions are represented by descriptive titles. LightWave has three separate programs. 'Modeler' is a program which handles all modeling functions, such as providing tools for creating 3D objects. 'Layout' is a program where all scene-building and animation functions such as lighting and animating occur. Within 'Layout' is a renderer program called ScreamNet.

The workflow for this project in LightWave would involve acquiring or using Modeler to create objects and store them under appropriate categories, loading objects into Layout, assigning surfaces and textures to objects, adding and scheming lights within scenes, animating scenes by generating key frames for objects, light and/or the camera, assigning resolution and camera attributes, and rendering and saving image sequences.

\section{Selecting a Modeling Tool}

One of the ongoing debates in computer animation is choosing what modeling tool to use, which is often an exercise that involves weighing which program is the most competitive, the fastest, or the one that has the most number of features. This is a difficult question to answer. New Tech's LightWave can be classified as the easiest 3D animation program to learn, but it may be shallow in terms of some of its facilities, especially where character animation is concerned. Discreet's 3ds Max is more difficult to learn than LightWave, but its robust features increase its depth. Maya sits at the top of the difficulty scale. It is difficult to learn and the features offered are complicated to comprehend. One needs to attend school to learn Maya and become a professional animator competent in Maya.

$3 \mathrm{~d}$ Max offers a good balance between depth of functionality and degree of difficulty. It offers great character creation tools and an enormous collection of third-party plug-ins that seem to be incorporated as a feature in its upgrades. Weighing the pros and cons, this project used 3ds Max for the character movement and environment modeling of the Street Skateboarding Simulation. 


\section{Character Modeling}

A good character model has to fulfill two requirements: It has to be visually appealing and it has to animate well. The aesthetic quality and technical functionality of the model are deeply intertwined. A character model and skeleton is a large and complex hierarchy. As such, problems that exist at or near a model's base will spread throughout the entire figure. Therefore, a comprehensive and step-by-step solution should be made to reduce as many changes as possible to the model's base construction. These changes can affect the entire process of character modeling improvements.

Before the modeling process begins, it is important to have reference drawings or character images. This is achieved through either creating sketches or collecting images that will help in building the model. As a minimum requirement, the modeler will need images from the front, side, and perspective. Depending on whether the modeler will need to illustrate other parts of the model, other images will probably be needed such as images of the back, underside, and top. Figure 6 shows an early development of the character model for this project.

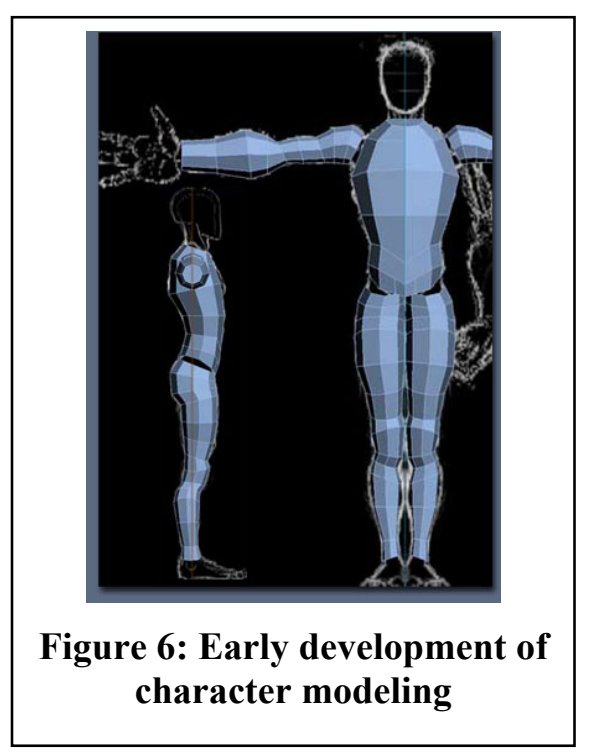

After the character model is complete, it will be given a set of animation controls (also known as a rig) which will facilitate easy and quick animation of the character model. Although building the rig will entail more time, this exercise will prove beneficial as the rig will offer significant help in making real movement animation. $3 \mathrm{ds}$ Max has a biped technique to make the rigging much easier - the process only requires a simple application to the view port and changes values on the "Create Biped" rollout into appropriate values for the character model mesh. Next is to move, resize, and rotate each part in the biped to fit inside the character model mesh.

According to Animation and Interactive Media (AIM), there are a number of tricks that animators constantly use to cut down the amount of work done. Cycles of repeating action are one of these ways; animators seek opportunities to include a cycle in a sequence.

Using the technique of cyclic animation, it is possible for animators to reuse a sequence of drawings many times over to build up screen time without additional effort. Some cycles may consist of only two drawings. Others may involve several complex actions.

Sequences can be cyclic in nature, that is, they can be ordered as $1,2,3,4$ followed by an exact repetition of that order as $1,2,3,4$, etc. This type of cycle is useful for representing things like a spinning wheel. In addition, cycles can be random, for example, 1,4,3,1,2,4,3,1,2, etc. This type of cycle is suitable when one wishes to mimic the motion of a flickering flag.

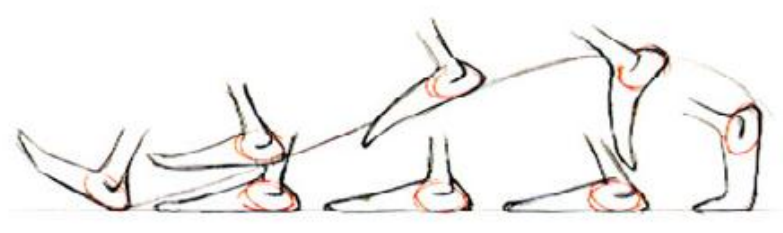

Figure 7: Typical Foot Action (Williams, 2002) 
This property of digital development could speed up development time compared to traditional movie making. For example, a typical foot action shown in Figure 7 could be reused for many purposes as long as it involves the movement of a human character.

The animation outputs are directed to movie files using AVI as the format since it is selfcontained and holds the entire image - a requirement for the animation to play back frames. However, the final product user interface is built with Adobe Flash (http://www.adobe.com/products/flashplayer/). Therefore, the output files have to be converted into FLV (Flash Video file format) that allows for higher compression with some loss of quality.

\section{System Design}

The final product fits into an $82 \mathrm{MB}$ space on a hard disk. This is considered a very small space, as a physically small-sized USB flash could have some Giga bytes of capacity these days. It could also fit onto a cheap storage device such as a CD which has around $700 \mathrm{MB}$ capacities. Aside from having a flash player in the viewer's computer, there is no installation required to view this simulation software. Although the ubiquity of a flash player in a computer would not become an obstacle for viewing the simulation software, the player itself could be obtained for free (http://get.adobe.com/flashplayer/?promoid=DXLUJ). Hence the Street Skateboarding Simulation is affordable for the target audience (aged $15-25$ ) in terms of price and portability.

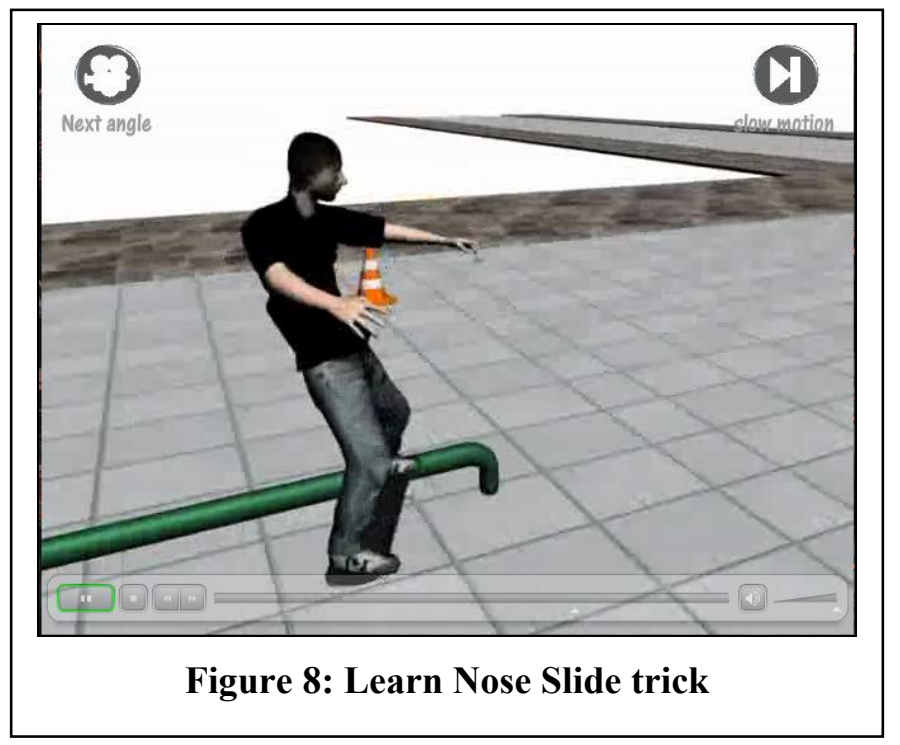

At the heart of its function, the software displays a menu enabling users to choose the trick video that they want to watch or to read the trick's instruction steps. Figures 8, 9, and 10, show an example of a 3D animation that may be studied when a user clicks the "Nose Slide" trick. The user interface includes normal video functions at the bottom of the movie such as fast forward, rewind, pause, play, stop, and a slide bar to interactively choose a particular frame.

At the top left there is a "Next Angle" button to move the camera to another given angle. In this case, the same scene which the user views (Figure 8) is zoomed resulting in the image shown in Figure 9. Similarly, the user can view the same scene from behind, as is shown in Figure 10.

The "Slow motion" button at the top right allows users to slow down the animation. 
Figure 8 shows a trick from one camera angle. A user might not be able to see the left leg clearly, and the part of the board used to slide along the pipe. Figure 9 shows the same object from another angle. From the angle, it can be seen that the wheel does not go to the other side of the iron bar. Another angle is provided in Figure 10, which confirms that only the tips of the skateboard slide along the iron bar.

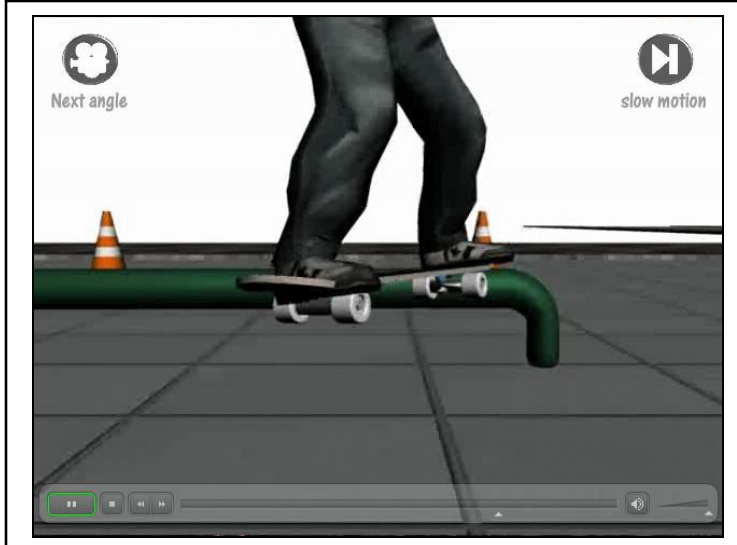

Figure 9: View a magnified Nose Slide trick

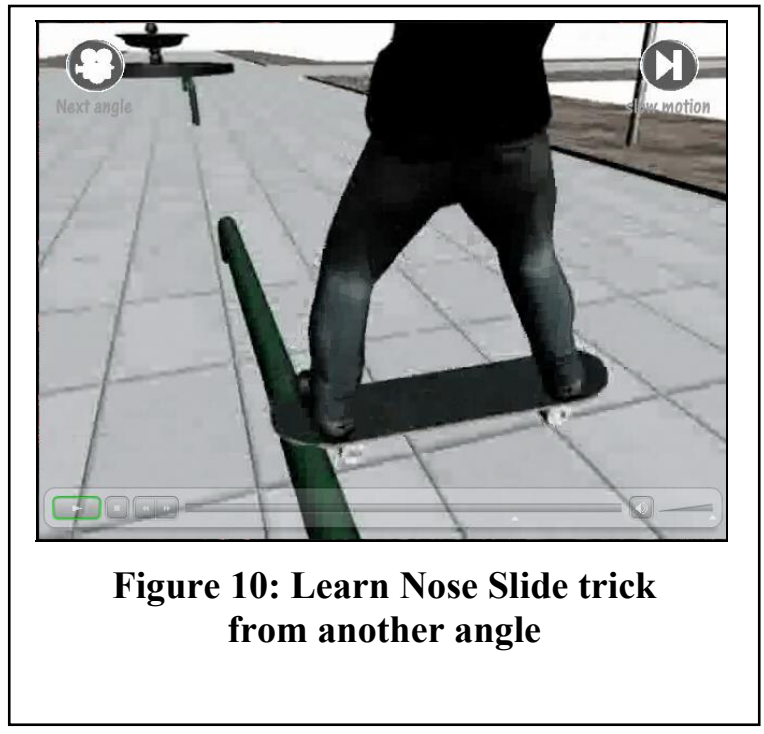

At the front part, the menu showing different tricks is displayed. Here, the user interface provides a menu which includes a voice play for written instructions. This allows the user to choose to either read the text or listen to the voice play when viewing the written instructions. This feature is easy to use when the target users are beginners.

The final implementation is installing a flash player on the computer. The pixel size required for the user interface would be $800 \times 600$ pixels, which is the conventional size of an ordinary flash application. This makes the scene viewable from any conventional screen.

\section{Evaluation}

In order to evaluate the usefulness of the Street Skateboarding Simulation, we conducted a user test by observing the target audience. Elements of the observation included how easy the users navigated and how frustrated they were when asked to accomplish a given task. An example of a task is, "You want to learn how to Ollie. Please show me how to display that from the interface."

The first attempt of the evaluation was impossible to accomplish when we went to a skateboard park and asked a volunteer. It was hard for the user to see an image on a laptop under the sun, worsened by a busy and noisy background. Henceforth the list of users was gathered from the skateboarder acquaintances of one of the authors. The user test also included non-skateboarders since the Street Skateboarding Simulation was to demonstrate the usefulness of having a new skateboarding learning medium (3D angle rotations) for beginners and prospects. Each of them was contacted for an appointment and the testing was conducted at a separate time at a convenient location.

There were 27 respondents in total. A questionnaire was provided after the interview (see the Results section). There were 24 male and 3 female users between 15 to 25 years of age. Users were briefed through the same procedure before testing was conducted, i.e., they were told that it was not an IQ test, and that the researchers were testing the software interface, not the users. This 
would avoid users rushing when navigating and give them a chance to reveal any honest expressions whether verbal or non-verbal.

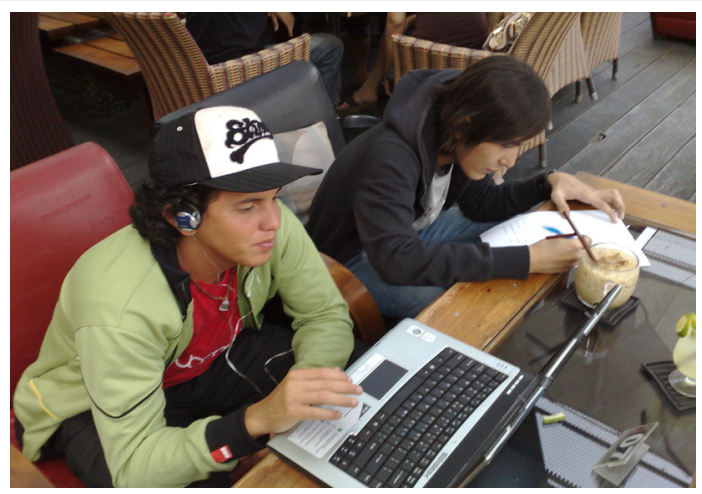

Figure 11: User testing the Street Skateboarding Simulation

The evaluation was conducted by allowing respondents to use a laptop and a pair of headphones so they could use the simulation free from external interference (Figure 11). Thereafter, the respondents were asked to complete a questionnaire which had multiple choice questions.

\section{Results}

Tables 1-13 show the results of the survey described in the previous section.

\begin{tabular}{|c|c|c|}
\hline \multicolumn{3}{|c|}{ Table 1: Screen layout was helpful } \\
\hline $\begin{array}{c}\text { Range (1=never, } \\
\text { 5=always) }\end{array}$ & $\begin{array}{c}\text { Fre- } \\
\text { quency }\end{array}$ & Percent \\
\hline 1 & 0 & 0 \\
\hline 2 & 0 & 0 \\
\hline 3 & 2 & 7.4 \\
\hline 4 & 18 & 66.7 \\
\hline 5 & 7 & 25.9 \\
\hline Total & 27 & 100.0 \\
\hline \multicolumn{3}{|c|}{ mean $=4.19$} \\
\hline s.d. & 0.28 & \\
\hline
\end{tabular}

Table 2: Color combinations used

\begin{tabular}{|c|c|c|}
\hline $\begin{array}{c}\text { Range }(1=\text { static }, \\
5=\text { dynamic })\end{array}$ & $\begin{array}{c}\text { Fre- } \\
\text { quency }\end{array}$ & Percent \\
\hline 1 & 0 & 0 \\
\hline 2 & 1 & 3.7 \\
\hline 3 & 5 & 18.5 \\
\hline 4 & 16 & 59.3 \\
\hline 5 & 5 & 18.5 \\
\hline Total & 27 & 100.0 \\
\hline \multicolumn{3}{|c|}{ mean $=3.93$} \\
\hline s.d. & 0.46 & \\
\hline
\end{tabular}




\begin{tabular}{|c|c|c|}
\hline \multicolumn{3}{|c|}{ Table 3: Amount of information } \\
\hline $\begin{array}{c}\text { Range } \\
\text { (1=inadequate, } \\
5=\text { adequate) }\end{array}$ & $\begin{array}{c}\text { Fre- } \\
\text { quency }\end{array}$ & Percent \\
\hline 1 & 0 & 0 \\
\hline 2 & 0 & 0 \\
\hline 3 & 2 & 7.4 \\
\hline 4 & 10 & 37.0 \\
\hline 5 & 15 & 55.6 \\
\hline Total & 27 & 100.0 \\
\hline \multicolumn{3}{|c|}{ mean $=4.48$} \\
\hline \multicolumn{3}{|c|}{ s.d. $=0.32$} \\
\hline
\end{tabular}

\begin{tabular}{|c|r|r|}
\hline \multicolumn{3}{|c|}{ Table 4: Sequence of screens } \\
\hline $\begin{array}{c}\text { Range } \\
\text { (1=confusing, } \\
\text { 5=clear) }\end{array}$ & $\begin{array}{c}\text { Fre- } \\
\text { quency }\end{array}$ & Percent \\
\hline 1 & 0 & 0 \\
2 & 0 & 0 \\
3 & 9 & 33.3 \\
4 & 11 & 40.7 \\
5 & 7 & 25.9 \\
& 27 & 100.0 \\
\hline \multicolumn{3}{|c|}{ Total } \\
mean $=3.93$ \\
\hline \multicolumn{3}{|c|}{ s.d. $=0.27$} \\
\hline \multicolumn{2}{|c|}{} \\
\hline
\end{tabular}

\begin{tabular}{|c|c|c|}
\hline \multicolumn{3}{|c|}{$\begin{array}{c}\text { Table 5: Going back to the previous } \\
\text { display page }\end{array}$} \\
\hline $\begin{array}{c}\text { Range } \\
\begin{array}{c}(1=\text { impossible } \\
5=\text { easy })\end{array}\end{array}$ & $\begin{array}{c}\text { Fre- } \\
\text { quency }\end{array}$ & Percent \\
\hline 1 & 0 & 0 \\
\hline 2 & 0 & 0 \\
\hline 3 & 5 & 18.5 \\
\hline 4 & 10 & 37.0 \\
\hline 5 & 12 & 44.4 \\
\hline Total & 27 & 100.0 \\
\hline \multicolumn{3}{|c|}{ mean $=4.26$} \\
\hline s.d. & 0.29 & \\
\hline
\end{tabular}

Table 6: Texts on the user interface

\begin{tabular}{|c|c|c|}
\hline $\begin{array}{c}\text { Range } \\
(1=\text { hard to read, } \\
5=\text { easy) }\end{array}$ & $\begin{array}{c}\text { Fre- } \\
\text { quency }\end{array}$ & Percent \\
\hline 1 & 0 & 0 \\
\hline 2 & 7 & 25.9 \\
\hline 3 & 7 & 25.9 \\
\hline 4 & 6 & 22.2 \\
\hline 5 & 7 & 25.9 \\
\hline Total & 27 & 100.0 \\
\hline \multicolumn{3}{|c|}{ mean $=3.48$} \\
\hline s.d. $=$ & .43 & \\
\hline
\end{tabular}

\begin{tabular}{|c|c|c|}
\hline \multicolumn{3}{|c|}{ Table 7: Command buttons } \\
\hline $\begin{array}{c}\text { Range } \\
\left(\begin{array}{c}1=\text { confusing, } \\
5=\text { clear })\end{array}\right.\end{array}$ & $\begin{array}{c}\text { Fre- } \\
\text { quency }\end{array}$ & Percent \\
\hline 1 & 0 & 0 \\
\hline 2 & 0 & 0 \\
\hline 3 & 8 & 29.6 \\
\hline 4 & 8 & 29.6 \\
\hline 5 & 11 & 40.7 \\
\hline Total & 27 & 100.0 \\
\hline \multicolumn{3}{|c|}{ mean $=4.11$} \\
\hline \multicolumn{3}{|c|}{ s.d. $=0.27$} \\
\hline
\end{tabular}

\begin{tabular}{|c|c|c|}
\hline \multicolumn{3}{|c|}{$\begin{array}{l}\text { Table 8: Length of delay between } \\
\text { operations }\end{array}$} \\
\hline $\begin{array}{c}\text { Range } \\
\left(\begin{array}{c}1=\text { unacceptable } \\
5=\text { unnoticed })\end{array}\right.\end{array}$ & $\begin{array}{l}\text { Fre- } \\
\text { quency }\end{array}$ & Percent \\
\hline 1 & 0 & 0 \\
\hline 2 & 1 & 3.7 \\
\hline 3 & 3 & 11.1 \\
\hline 4 & 14 & 51.9 \\
\hline 5 & 9 & 33.3 \\
\hline Total & 27 & 100.0 \\
\hline \multicolumn{3}{|c|}{ mean $=4.15$} \\
\hline s.d. $=$ & .50 & \\
\hline
\end{tabular}




\begin{tabular}{|c|c|c|}
\hline \multicolumn{3}{|c|}{$\begin{array}{l}\text { Table 9: Getting started with } \\
\text { the simulation }\end{array}$} \\
\hline $\begin{array}{c}\text { Range (1=difficult, } \\
5=\text { easy) }\end{array}$ & $\begin{array}{c}\text { Fre- } \\
\text { quency }\end{array}$ & Percent \\
\hline 1 & 0 & 0 \\
\hline 2 & 0 & 0 \\
\hline 3 & 1 & 3.7 \\
\hline 4 & 11 & 40.7 \\
\hline 5 & 15 & 55.6 \\
\hline Total & 27 & 100.0 \\
\hline \multicolumn{3}{|c|}{ mean $=4.52$} \\
\hline s.d. $=$ & 32 & \\
\hline
\end{tabular}

\begin{tabular}{|c|r|r|}
\hline \multicolumn{3}{|c|}{$\begin{array}{c}\text { Table 10: Response time } \\
\text { for most operations }\end{array}$} \\
\hline $\begin{array}{c}\text { Range (1=slow, } \\
\text { 5=fast) }\end{array}$ & $\begin{array}{c}\text { Fre- } \\
\text { quency }\end{array}$ & Percent \\
\hline 1 & 0 & 0 \\
2 & 0 & 0 \\
3 & 4 & 14.8 \\
4 & 14 & 51.9 \\
5 & 9 & 33.3 \\
& 27 & 100.0 \\
\hline \multicolumn{3}{|c|}{ Total } \\
mean $=4.19$ \\
\hline \multicolumn{3}{|c|}{ s.d. $=0.28$} \\
\hline
\end{tabular}

\begin{tabular}{|c|c|c|}
\hline \multicolumn{3}{|c|}{$\begin{array}{l}\text { Table 11: Quality of still pictures } \\
\text { (size, brightness, color) }\end{array}$} \\
\hline $\begin{array}{l}\text { Range }(1=\text { bad, } \\
5=\text { good })\end{array}$ & $\begin{array}{l}\text { Fre- } \\
\text { quency }\end{array}$ & Percent \\
\hline 1 & 0 & 0 \\
\hline 2 & 1 & 3.7 \\
\hline 3 & 8 & 29.6 \\
\hline 4 & 12 & 44.4 \\
\hline 5 & 6 & 22.2 \\
\hline Total & 27 & 100.0 \\
\hline \multicolumn{3}{|c|}{ mean $=3.85$} \\
\hline \multicolumn{3}{|c|}{ s.d. $=0.45$} \\
\hline
\end{tabular}

Table 12: Quality of animation (size, brightness, color)

\begin{tabular}{|c|c|c|}
\hline $\begin{array}{c}\text { Range }(1=\text { bad, } \\
5=\text { good })\end{array}$ & $\begin{array}{c}\text { Fre- } \\
\text { quency }\end{array}$ & Percent \\
\hline 1 & 0 & 0 \\
\hline 2 & 3 & 11.1 \\
\hline 3 & 8 & 29.6 \\
\hline 4 & 12 & 44.4 \\
\hline 5 & 4 & 14.8 \\
\hline Total & 27 & 100.0 \\
\hline \multicolumn{3}{|c|}{ mean $=3.63$} \\
\hline \multicolumn{3}{|c|}{ s.d. $=0.43$} \\
\hline
\end{tabular}

\begin{tabular}{|c|c|c|}
\hline \multicolumn{3}{|c|}{ Table 13: Sound output (voice play) } \\
\hline $\begin{array}{l}\text { Range }(1=\text { hard to } \\
\text { hear, } 5=\text { audible })\end{array}$ & $\begin{array}{c}\text { Fre- } \\
\text { quency }\end{array}$ & Percent \\
\hline 1 & 0 & 0 \\
\hline 2 & 0 & 0 \\
\hline 3 & 7 & 25.9 \\
\hline 4 & 12 & 44.4 \\
\hline 5 & 8 & 29.6 \\
\hline Total & 27 & 100.0 \\
\hline \multicolumn{3}{|c|}{ mean $=4.04$} \\
\hline \multicolumn{3}{|c|}{ s.d. $=0.27$} \\
\hline
\end{tabular}

\section{Discussion}

No difficulty was observed whilst the users explored the user interface. There was no frustration or resentment that showed dissatisfaction when the users navigated the software.

In general, it could be said that the Street Skateboarding Simulation was well received by $93 \%$ of the respondents as a good alternative to learn the basics of street skateboarding. The software displayed a number of difficult tricks which require advance skateboarding skills and many hours of 
practice. The software's ability to move the camera, allowing the user to view the tricks from different angles, excited $67 \%$ of them, while $22 \%$ of respondents who enjoyed the angle movement provided some criticism of the software. On the other hand, the software failed to catch the attention of $11 \%$ of the remaining users.

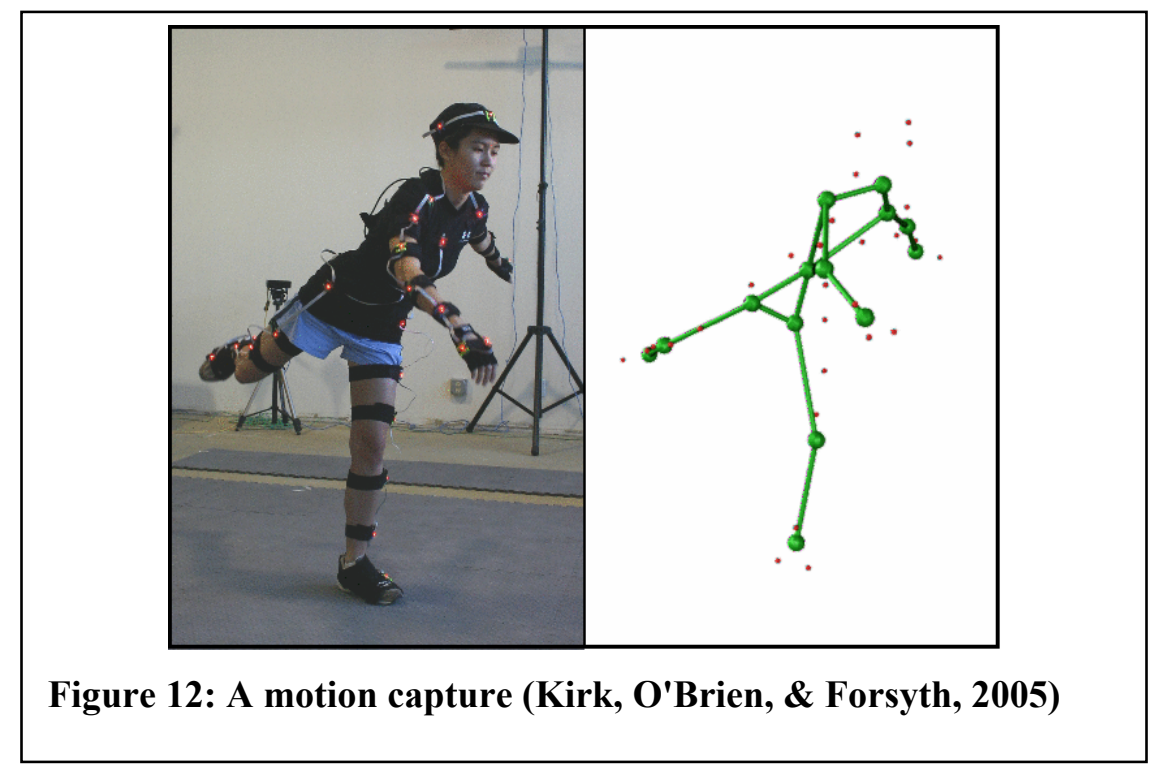

Critique of 3D videos was directed at movements which were not smooth (Table 12). Given a scale of 1 (bad) to 5 (best), only $15 \%$ rated the videos with a 5 while $45 \%$ rated them with a 4 . $30 \%$ gave a rating of 3 , and the remaining $10 \%$ gave the software a rating of 2 . The respondents further said that foot positions are not clear although they stand at the right place when examined in detail. The reason for this is that it is difficult to render a realistic 3D animation using standalone features from the development tool described above. It may take a very long time to imitate real human movements without motion capture equipment as seen in Figure 12. At the time of writing, only one commercial company owned such gear in Indonesia, the country where this study took place.

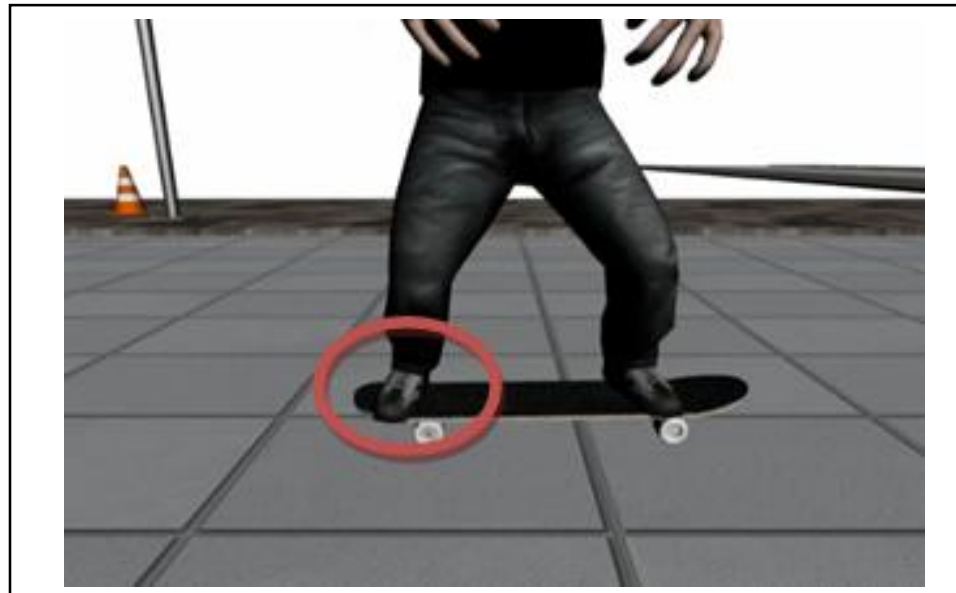

Figure 13: The indication point while the speaker must explain the steps clearly and briefly
Compared to traditional skateboarding videos, about $74 \%$ of the respondents thought that the simulation is more helpful to learn from. That number could be higher as its validity is questionable. Some of the respondents who said "no" wished to have more advanced tricks. Furthermore, some users preferred to have control on the speed of the slow motion feature in order to make this simulation much more useful than a normal video.

One notable suggestion is to combine the $3 \mathrm{D}$ animation, a 
voice play that explains how to do the skateboard trick step-by-step, and a pointer such as that shown in Figure 13 that emphasizes which foot position is required for a particular trick, and the ability to change the view angle interactively according to what is being explained.

Finally, one encouraging response was that the software has the potential to further educate people who do not know anything about skateboarding, with the end goal of convincing them to take up skateboarding as a hobby or sport.

\section{Conclusions and Future Work}

Using the Street Skateboarding Simulation as a measuring tool, it was shown that 3D simulations with angle rotation capabilities provide a more effective way of learning physical skills compared to other available means such as videos or books. It is a more cost-efficient solution compared to having private lessons or watching an expert in real time.

\section{The Skateboarding Simulation Software}

Future developments could incorporate more detailed and smooth movements using motion capture equipment, which can record the movements of a professional skateboarder. Some of the respondents stated that they look forward to the next version of the software where all suggestions have been addressed. A range of tricks at the beginner, amateur, and professional levels would attract more of an audience and increase the usability of the software. With respect to those, a study that observes movement patterns for a specific activity would enhance the development of this type of software by using reusable modules.

It can be argued that a smooth 3D simulation that can be moved, rotated, and magnified interactively allowing users to observe movement from any angle and precise timing of skateboarding tricks would revolutionize physical-based learning.

\section{A Study on the Learning Effectiveness}

One part of the effort has been devoted to improve the human-computer interaction of the software. The other part was to test learning effectiveness through a "within subjects" experimental design where each user learned from the proposed medium and compared his learning experience with traditional media. The result was a subjective opinion from $74 \%$ of the users who said that the software gave them a better learning experience. A larger study in terms of participant numbers and a longer study in terms of participation time span are required to provide empirical data on the efficiency of the simulation software approach compared to the traditional more established approaches.

The challenge to this is that individual learning curve naturally varies - some may take months to accomplish the first trick while talented ones may require only a couple of tries. Hence we suggest a "between subjects" experimental design as a future work where each group only learns from one medium which is different from the other. This approach will require a large number in each group, for the purpose of having a reliable internal validity to endure that the observed result is actually caused by the learning medium used.

To increase the reliability of its external validity, it is suggested that the experiment include other types of sports. As any sports or games have tricks that would take time for novices to learn, further work on this study should design and implement a simulation software application for a wider range of sports and test its effectiveness in improving the game. 


\section{References}

Aldrich, C. (2005). Learning by doing: A comprehensive guide to simulations, computer games, and pedagogy in e-learning and other educational experiences. San Fransisco: Pfeiffer.

Autodesk 3ds Max. (n.d.). In Wikipedia, The Free Encyclopedia. Retrieved April 26, 2008, from http://en.wikipedia.org/wiki/3ds_max

Autodesk Maya. (n.d.). In Wikipedia, The Free Encyclopedia. Retrieved April 23, 2008, from http://en.wikipedia.org/wiki/Autodesk_Maya

Cave, S. (n.d.). Top 6 skateboarding games. Retrieved May 3, 2008, from http://skateboard.about.com/od/games/tp/Skateboarding_Games.htm

Connolly, T., \& Stansfield, M. (2006). Using game-based eLearning technologies in overcoming difficulties in teaching information systems. Journal of Information Technology Education, 5, 459476. Retrieved from http://www.jite.org/documents/Vol5/v5p459-476Connolly170.pdf

Goodman, D., Bradley, N. L., Paras, B., Williamson, I. J., \& Bizzochi, J. (2005). Video gaming promotes concussion knowledge acquisition in youth hockey players. Journal of Adolescence, 29(3), 351-360.

Greasley, A. (2008). Enabling a simulation capability in the organisation. London, UK: Springer-Verlag.

Gregory, S., Greatrex, N., Timms, D., Gaddum, N., Pearcy, M., \& Fraser, J. (2010). Simulation and enhancement of a cardiovascular device test rig. Journal of Simulation, 4, 34-41.

HighwayHome. (n.d.). Sports simulation games \& sim leagues. Retrieved September 9, 2009, from http://www.highwayhome.com/sports/sports_simulations/sports_sims_games.html

Kirk, A., O'Brien, J., \& Forsyth, D. (2005). Skeletal parameter estimation from optical motion capture data. Computer Vision and Pattern Recognition, 2005. CVPR 2005. IEEE Computer Society Conference on (pp. 782-788). IEEE XPlore.

Klassen, K. J., \& Willoughby, K. A. (2003). In-class simulation games: Assessing student learning. Journal of Information Technology Education, 2, 1-13. Retrieved from http://www.jite.org/documents/Vol2/v2p001-013-59.pdf

Lightwave 3D. (n.d.). In Wikipedia, The Free Encyclopedia. Retrieved April 26, 2008, from http://en.wikipedia.org/wiki/LightWave_3D

PrimeTime Amusements. (n.d.). Top Skater: PrimeTime amusements video arcade games. Retrieved September 9, 2009, from PrimeTime Amusements: Video Arcade Games Machine Sales and Rentals: http://www.primetimeamusements.com/arcadegame.php?id=150

Reed Business Information. (2009). Digital instructors: Simulation software helps production workers learn faster. Retrieved September 9, 2009, from Manufacturing Business Technology: http://www.mbtmag.com/article/192696Digital_instructors_Simulation_software helps production_workers learn faster.php

Shaffer, D. W. (2006). How computer games help children learn. New York: Palgrave Macmillan.

Solomon, M. R. (2004). Consumer behavior: Buying, having, and being ( $6^{\text {th }}$ ed.). Upper Saddle River, New Jersey: Pearson Education, Inc.

Williams, R. (2002). The animator's survival kit. London, UK: Faber \& Faber Limited.

Wysaski, J. (n.d.). How does skateboarding rank in popularity compared to other sports? Retrieved June 27, 2008, from eHow: http://www.ehow.com/how-does 4580013 rank-popularity-compared-othersports.html 


\section{Biographies}

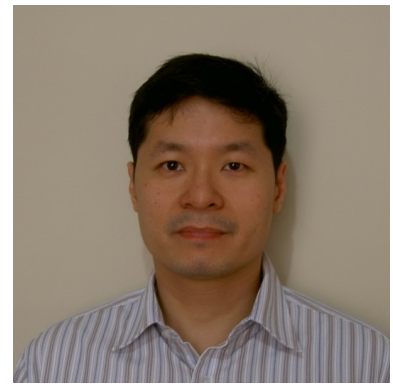

Erwin Adi is a lecturer at BINUS International - BINUS University, Jakarta, Indonesia, where he teaches Web Programming and User Interface Engineering classes. His early career includes working as a Network Engineer with several telecom companies such as British Telecom in Belgium. During this time, he gained experience in handling fiber network, controlling European-wide network under a wide range of platform, troubleshooting IP-related problems, and mitigating high-impact network failures. Erwin has a Master degree in Telecommunications from The University of Strathclyde, Glasgow, UK. His Bachelor degree was in Computer Science and Applied Mathematics/Statistics (double major) from The State University of New York at Stony Brook, USA, where he received Dean's Lists for 3 semesters, and was called in to join the Sigma Beta Honor Society.

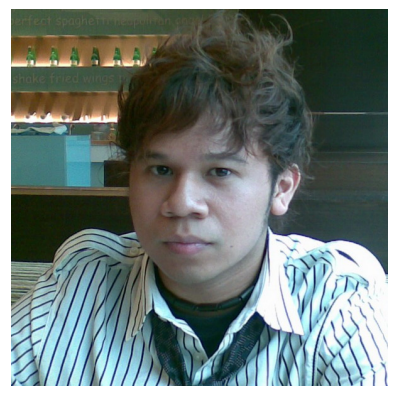

I Gde Made Krisna Aditya, also called Adit, works in Mizuho Bank in Business Development Department. He earned a Bachelor degree in Computer Science from BINUS International - BINUS University, Jakarta, Indonesia, with a minor in Marketing. Adit is multicultural and is passionate in learning foreign languages such as Japanese. He is also an avid street skateboarder, and he has skateboarded with his friends wherever his life brings him. His observations while skateboarding in Paris, France and in Bandung, Indonesia have inspired him to combine his knowledge in human-computer interface and his hobby into an educational medium for skateboarders. He dedicates this work as an appreciation for his skateboarding friends who kindly taught him skateboarding tricks.

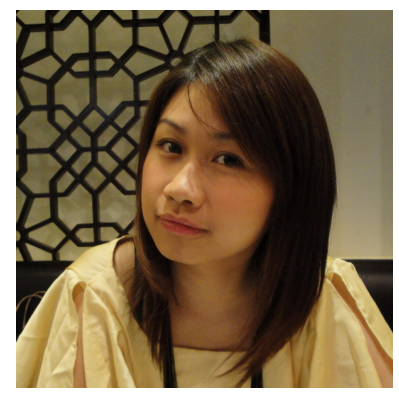

Meriyana Citrawati is an Assistant to the Project Manager at Citibank Indonesia. She graduated from BINUS International - BINUS University, Jakarta, Indonesia with a Bachelor degree in Computer Science, focusing in Multimedia technology and development. She was especially interested in developing the modeling of 3D character animations during her study where she spent a lot of time building the models from all angles. One part that she enjoyed was receiving comments and critics when she tested her work to the open public, as it allowed her to learn how to optimize the models. 